\title{
Improvement of LMS adaptive noise canceller using uniform Poly-phase digital filter bank
}

\author{
Alaa Hadi Mohammad, Azura Che Soh, Noor Faezah Ismail, Ribhan Zafira Abdul Rahman, \\ Mohd Amran Mohd Radzi \\ Control System \& Signal Processing (CSSP) Research Centre, Department of Electrical and Electronic Engineering, \\ Faculty of Engineering, Universiti Putra Malaysia
}

\begin{tabular}{l} 
Article Info \\
Article history: \\
Received Jun 22, 2019 \\
Revised Sep 24, 2019 \\
Accepted Oct 7, 2019 \\
\hline Keywords: \\
Decomposition technique \\
Least mean square (LMS) \\
algorithm filter bank \\
Noise canceller \\
Poly-phase digital filt fourier transform \\
discrete \\
(DFT)
\end{tabular}

\begin{abstract}
This paper presents the Least Mean Square (LMS) noise canceller using uniform poly-phase digital filter bank to improve the noise can-cellation process. Analysis filter bank is used to decompose the full-band distorted input signal into sub-band signals. Decomposition the full-band input distorted signal into sub-band signals based on the fact that the signal to noise ratio $(\mathrm{S} / \mathrm{N})$ is inversely proportional to the signal bandwidth. Each subband signal is fed to individual LMS algorithm to produce the optimal subband output. Synthesis filter bank is used to compose the optimal sub-band outputs to produce the final optimal full-band output. In this paper, m-band uniform Discrete Fourier Transform (DFT) digital filter bank has been used because its computational complexity is much smaller than the direct implementation of digital filter bank. The simulation results show that the proposed method provides the efficient performance with less and smooth error signal as compared to conventional LMS noise canceller.
\end{abstract}

Copyright @ 2020 Institute of Advanced Engineering and Science. All rights reserved.

Corresponding Author:

Azura Che Soh,

Department of Electrical and Computer Engineering,

Universiti Putra Malaysia,

43300 UPM Serdang, Selangor, Malaysia.

Email: azuracs@upm.edu.my

\section{INTRODUCTION}

The In the adaptive noise cancellers, least mean square (LMS) algorithm is widely used due to its extreme simplicity [1-5]. The concept of decomposition of the full-band signal into a number of sub-band signals is recently adopted to improve the convergence rate of the least mean square adaptive filter. Decomposition of full-band signal into a number of sub-band signals greatly reduces the noise level in the output signal and increases the update rate of the adaptive filter [6-8]. In addition, using decomposition of the full-band input signal into sub-band input signals in the adaptive cancellation systems will reduce the computational expenses in design of these systems [2, 9-10].

A number of sub-bands adaptive filtering methods have been proposed to improve the adaptation process [11-15]. In critical sample filtering method, the presence of aliasing distortions requires the use of adaptive cross filter between sub-bands [16] as long as the distorted input signal is not band-limited signal [17]. The adaptive systems that use cross adaptive filters generally converge slowly and have high computational expenses [18-19]. With gap filter banks method, a significant signal distortion is produced. To avoid aliasing distortion associated with the use of critical sample filtering, oversampled filter banks method has been proposed [20-21]. This method needs higher computational requirements than critical sample filtering method.

In addition, the oversampled filter banks themselves color the input signal. These problems can be related to the fact that oversampled sub-band inputs will generate an unstable correlation matrix [22] which 
leads to the divergence from the optimal output. A pre-emphasis filter for each sub-band is suggested [23] to treat the slow convergence in the systems that use the cross adaptive filter method; however this method has a complexities and limitations in its implementation such as power dissipation and cost.

The aim of this paper is to improve the performance of least mean square LMS adaptive filter. This improvement is done by decomposition the full-band input signal into sub-band input signals by using polyphase digital filter bank [24-25] to reduce the noise level that associated with the input signal.

The paper is organized as follows. In the next section, the general algorithm of the Poly-phase Digital Filter Bank is described. Section 3 briefly discusses the general technique of LMS Adaptive noise canceller system. Section 4 discusses the implementation of proposed technique for noise canceller system. The technique is Improved LMS Adaptive Noise Canceller (ILMS) is implemented by using uniform DFT digital filter bank, based on decomposition of the full-band distorted signal into sub-band signals. Section 5 discusses the performance of the proposed ILMS and compared with existing of LMS Adaptive noise canceller system. The conclusion of this paper is summarized in the last section.

\section{POLY-PHASE DIGITAL FILTER BANK}

Poly-phase digital filter bank consists of analysis filter bank and synthesis filter bank. Analysis filter bank is used to decompose the noisy full-band signal into improved sub-band signals. Synthesis filter bank is used to compose the improved sub-band signals into improved full-band signal.Tables and Figures are presented center, as shown below and cited in the manuscript.

\subsection{Analysis Filter Bank}

The sub-filters $H_{k}(z)$ in the analysis filter bank are the analysis filters. This filter is used to decompose the full-band discrete input signal $\mathrm{x}[\mathrm{n}]$ into sub-band discrete signals $y_{k}[n]$ that has a portion from the input full-band. Hence, let $H_{0}(z)$ represent a causal low-pass digital filter with real impulse response $h_{0}[n]$ then:

$$
H_{0}(z)=\sum_{n=\infty}^{\infty} h_{0}[n] z^{-n}
$$

The filter $H_{0}(z)$ is assumed to be Infinite Impulse Response (IIR) without any loss. Assume this filter has a pass-band edge frequency $\omega_{p}$ and stop-band edge frequency $\omega_{s}$ around $\pi / \mathrm{m}$, where $\mathrm{m}$ is some arbitrary integer. Hence, consider the transfer function $H_{k}(z)$ of the causal low-pass digital filter whose impulse response is $h_{k}(n)$ such that:

$$
h_{k}[n]=h_{0}[n] e^{\frac{j 2 \pi k n}{\mathrm{~m}}}=h_{0}[n] W_{\mathrm{m}}^{-k n}
$$

where $W_{\mathrm{m}}=e^{\frac{-j 2 \pi}{\mathrm{m}}}, \mathrm{m}$ discrete Fourier transfer function (DFT), thus:

$$
H_{k}(z)=\sum_{n=\infty}^{\infty} h_{k}[n] z^{-n}=\sum_{n=\infty}^{\infty} h_{0}[n] z^{-n} W_{\mathrm{m}}^{-k n}=\sum_{n=\infty}^{\infty} h_{0}[n]\left(z W_{m}^{k}\right)^{-n}
$$

where $\mathrm{k}=0,1, \ldots, \mathrm{m}-1$

As shown in (3) can be written as:

$$
H_{k}(z)=H_{0}\left(z W_{\mathrm{m}}^{k}\right), \mathrm{k}=0,1, \ldots, \mathrm{m}-1
$$

Then, the corresponding frequency response is:

$$
H_{k}\left(e^{j \omega}\right)=H_{0}\left(e^{j\left(\omega-\frac{2 \pi k}{\mathrm{~m}}\right)}\right), \mathrm{k}=0,1, \ldots, \mathrm{m}-1
$$

As shown in (5) states that the frequency response of $H_{k}(z)$ is obtained by shifting the response of $H_{0}(z)$ uniformly to the right-hand by $2 \pi \mathrm{k} / \mathrm{m}$. Therefore, this analysis filter bank is said to be uniform analysis filter bank. Now, the low-pass filter transfer function $H(z)$ can be represented in its even and odd coefficients. Hence, for 2-band $(\mathrm{m}=2)$ poly phase filter.

$$
H(z)=\sum_{n=0}^{\infty} h[2 n] z^{-2 n}+z^{-1} \sum_{n=0}^{\infty} h[1+2 n] z^{-2 n}
$$


Or,

$H(z)=E_{0}\left(z^{2}\right)+z^{-1} E_{1}\left(z^{2}\right)$

where:

$E_{0}(z)=\sum_{n=0}^{\infty} h[2 n] z^{-n}, E_{1}(z)=\sum_{n=0}^{\infty} h[1+2 n] z^{-n}$

Hence $E(z)$ can be generalized for m-band to be,

$E_{\ell}(z)=\sum_{n=0}^{\infty} h[\ell+\mathrm{m} n] z^{-n}, \ell=0,1, \ldots, \mathrm{m}-1$

Therefore,

$H(z)=\sum_{\ell=0}^{\mathrm{m}-1} z^{-\ell} E_{\ell}\left(z^{\mathrm{m}}\right)$

And thus:

$H_{0}(z)=\sum_{\ell=0}^{\mathrm{m}-1} z^{-\ell} E_{\ell}\left(z^{\mathrm{m}}\right)$

Substitute $z=z W_{\mathrm{m}}^{k}$ in (10) and use (4) yield,

$H_{k}(z)=\sum_{\ell=0}^{\mathrm{m}-1} z^{-\ell} W_{\mathrm{m}}^{-k \ell} E_{\ell}\left(z^{\mathrm{m}} W_{\mathrm{m}}^{k \mathrm{~m}}\right)$

But the identity $W_{\mathrm{m}}^{k \mathrm{~m}}=1$ with $\mathrm{k}=0,1, \ldots, \mathrm{m}-1$, then,

$H(z)=\sum_{\ell=0}^{\mathrm{m}-1} z^{-\ell} W_{\mathrm{m}}^{-k \ell} E_{\ell}\left(z^{\mathrm{m}}\right), k=0,1, \ldots, \mathrm{m}-1$

As shown in (12) can be written in matrices form a,

$\left[\begin{array}{c}H_{0}(z) \\ H_{1}(z) \\ H_{2}(z) \\ \vdots \\ H_{(\mathrm{m}-1)}(z)\end{array}\right]=\left[\begin{array}{ccccc}1 & 1 & 1 & \cdots & 1 \\ 1 & W_{\mathrm{m}}^{-1} & W_{\mathrm{m}}^{-2} & \cdots & W_{\mathrm{m}}^{-(\mathrm{m}-1)} \\ 1 & W_{\mathrm{m}}^{-2} & W_{\mathrm{m}}^{-4} & \cdots & W_{\mathrm{m}}^{-2(\mathrm{~m}-1)} \\ \vdots & \vdots & \vdots & \ddots & \vdots \\ 1 & W_{\mathrm{m}}^{-(\mathrm{m}-1)} & W_{\mathrm{m}}^{-2(\mathrm{~m}-1)} & \cdots & W_{\mathrm{m}}^{-(\mathrm{m}-1)^{2}}\end{array}\right]\left[\begin{array}{c}E_{0}\left(z^{m}\right) \\ z^{-1} E_{1}\left(z^{m}\right) \\ z^{-2} E_{2}\left(z^{m}\right) \\ \vdots \\ z^{-(m-1)} E_{m-1}\left(z^{m}\right)\end{array}\right]$

Then, by matrices notation as shown in (13) will be written as

$\mathrm{H}=\mathrm{DE}$

where: $\mathrm{H}$ is the $\mathrm{k}$-th filter matrix for obtaining $\mathrm{k}$-th sub-band transfer functions of full-band transfer function, $\mathrm{D}$ is $\mathrm{m} \times \mathrm{m}$ discrete Fourier transfer function (DFT) and $\mathrm{E}$ is the $\ell$-th poly-phase component of the filter matrix $\mathrm{H}$.

\subsection{Synthesis Filter Bank}

Synthesis filter bank is used to compose the sub-band signals which are produced from the analysis filter bank into full-band signal. Hence, from (14) the synthesis filter bank will be,

$\mathbf{E}=\mathbf{D}^{-1} \mathbf{H}$

where $\mathbf{D}^{\mathbf{- 1}}$ is the inverse of the DFT matrix (IDFT).

Figure 1 shows the implementation of the uniform DFT analysis and synthesis filters bank. The uniform DFT filter bank implementation is efficient and its computational complexity is much smaller than 
the direct implementation because the implementation of $\mathrm{m}$-band uniform DFT filter bank of N-tap low-pass filter requires a total of $\left(\frac{\mathrm{m}}{2} \log _{2} \mathrm{~m}+\mathrm{N}\right)$ multipliers, while the direct implementation requires (Nm) multipliers.

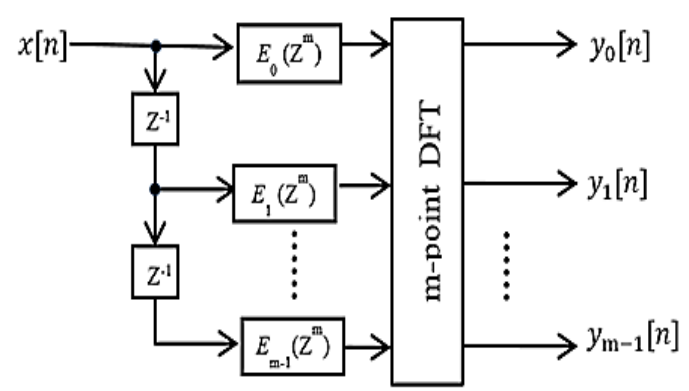

(a)

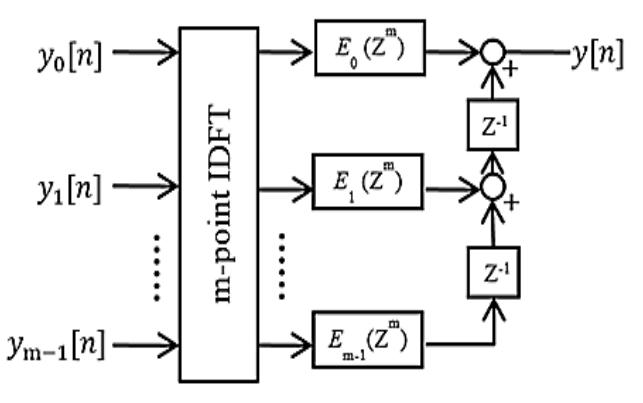

(b)

Figure 1. (a) Analysis filter bank and (b) Synthesis filter bank

\section{LMS ADAPTIVE NOISE CANCELLER}

LMS adaptive noise canceller adjusts the coefficients of the adaptive system to minimize the error between the desired signal and the error signal. LMS adaptive noise canceller involves simple computational resources and simple implementation but it is slowly converges from the optimal output. The weights update equation of the LMS adaptive noise canceller is given as:

$$
\mathbf{W}(n+1)=\mathbf{W}(n)+\mu e(n) \mathbf{X}(n)
$$

where: $\mu$ is a fixed step size of the adaptive system and it is used for control the convergence speed, $W(n)$ and $W(n+1)$ is the vectors weights at the instants ' $n$ ' and ' $n+1$ ' respectively, e(n) is the error between the desired signal and the output signal and $\mathrm{X}(\mathrm{n})$ is the input signal. The boundaries of the step size $\mu$ is given as:

$$
0<\mu<\frac{2}{\lambda_{\max }}
$$

Where $\lambda_{\max }$ is the maximum Eigen value of the input correlation matrix. These boundaries will limit the misadjustment M of the LMS adaptive noise canceller which is given by:

$$
M=\frac{\mu L \sigma_{x}^{2}}{2}
$$

where $\mathrm{L}$ is the number of Eigen values and $\sigma_{x}^{2}$ is the power of the input signal [1].

Misadjustment is a standard of the LMS adaptive noise canceller performance. Therefore, it should be kept as small as possible to reduce the excess in the mean square error at the steady-state. Hence from (18) it is noticed that larger step size for larger convergence speed, larger misadjustment and thus larger excess in the mean square error.

In this paper, the decomposition technique of the full-band distorted input signal into sub-band input signals is proposed to reduce the noise level associated with the input signal based on the fact that the noise level of a noisy signal directly proportional with the bandwidth of this signal. This decomposition is done by the analysis digital filter bank that has been derived above. Each sub-band signal that has low level of noise is fed to an individual LMS algorithm to minimize the error between the desired signal and the error signal. The aim of this decomposition process is to reduce the noisy adaptation process which is caused by the noisy gradient of the instantaneous mean square error that directly proportional with the noise level.

\section{IMPROVED LMS ADAPTIVE NOISE CANCELLER (ILMS)}

The performance improvement of the LMS adaptive noise canceller which is implemented by using uniform DFT digital filter bank, based on decomposition of the full-band distorted signal into sub-band signals. This decomposition will greatly reduce the level of the noise density associated with the desired

Improvement of LMS adaptive noise canceller using uniform Poly-phase digital... (Alaa Hadi Mohammad) 
signal. The purpose of this process is to reduce the noisy gradient of the instantaneous mean square error in the LMS noise canceller that increases with increasing the bandwidth of the noisy input signal. The decomposition of the full-band noisy signal into sub-band signals is based on the fact that the signal to noise ratio is inversely proportional to the bandwidth of this signal. The decomposition process will be done to both input distorted signal and desired signal to implement a synchronized adaptation process according to these sub-band signals.

Therefore, the sub-band signals carry a noise level smaller than the noise level of the input full-band distorted signal. This reduction in the noise level will increase the convergence speed of the LMS adaptive algorithm since it will reduce the noisy gradient of the instantaneous least mean square error. Hence, each sub-band signal will be fed to individual LMS adaptive algorithm to reduce the noisy adaptation process.

Figure 2 shows the implementation of the improved LMS adaptive noise canceller where it has been constructed by using the uniform analysis and synthesis filters bank of $m=3$ where the optimal output has been composed from the improved sub-band signals. In Figure 2: $x[n]$ is the input distorted signal, $x_{0}[n] x_{1}[n]$ and $x_{2}[n]$ are the sub-band signals of the input full-band signal $x[n], d[n]$ is the desired signal, $w_{0} w_{1}$ and $w_{2}$ are the weights that are updated by the LMS algorithm, $e_{0} e_{1}$ and $e_{2}$ are the error signals between the desired sub-band signals $d_{0}[n] d_{1}[n]$ and $d_{2}[n]$ of the desired full-band signal $d[n]$ and the improved sub-band signals $\hat{y}_{0}[n] \hat{y}_{1}[n]$ and $\hat{y}_{2}[n]$ of the improved full-band output signal $y[n]$ respectively.

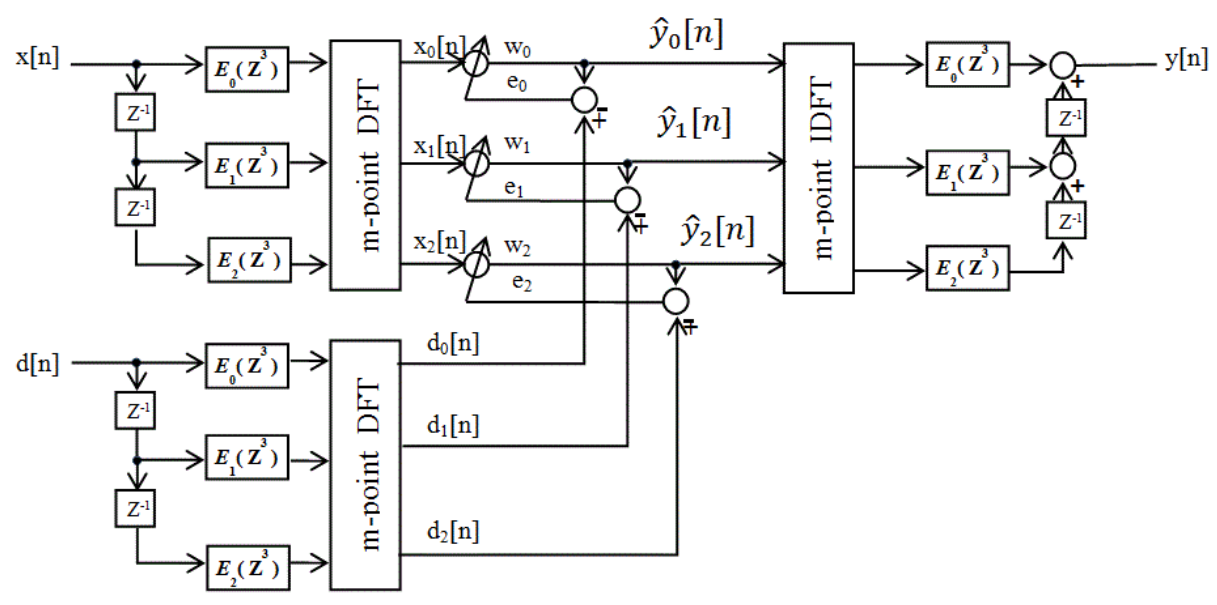

Figure 2. The proposed structure of the ILMS adaptive noise canceller

\section{RESULTS AND DISCUSSION}

In the MATLAB simulation of ILMS adaptive noise canceller, a sinusoidal wave form was distorted by a band-limited white noise which has been passed through a uniform analysis filter bank to decompose its full-band into three sub-band signals. By each sub-band signal, the density of the white noise that associated with the sinusoidal signal will be reduced according to the inverse proportion between the signal to noise ratio and the bandwidth of this signal as shown in Figure 3.

Figure 4 shows a comparison between the output signals and the error signals of the ILMS adaptive noise canceller and the LMS adaptive noise canceller. The input signal that has been used in this simulation is sinusoidal signal with $2 \mathrm{Vpp}$ and frequency of $2(\mathrm{rad} / \mathrm{sec})$ distorted by a band-limited white noise. The ILMS has shown results as compared to the LMS adaptive noise canceller.

Figure 5 compares between the graphs of the relationship between the mean square error (MSE) of the LMS adaptive noise canceller and the mean square error (MSE) of the ILMS noise canceller via the number of stages (number of sub-bands $m$ for ILMS adaptive noise canceller and canceller length L for LMS adaptive noise canceller) for different step size $\mu$. From Figure 5 it is noticed that with increasing the step size $\mu$, the convergence speed will increase without increasing the misadjustment. Therefore, this method will solve the direct proportion problem between the step size $\mu$ and the misadjustment $M$ that appears in (19). In addition, the number of the adaptation stages will be reduced such that the output MSE of the LMS adaptive noise canceller with $\mathrm{L}=10$ is $0.007 \mathrm{~V}$, while with the same output MSE of the ILMS adaptive noise canceller $\mathrm{m}=8$ as shown in Tables 1 and 2 . 


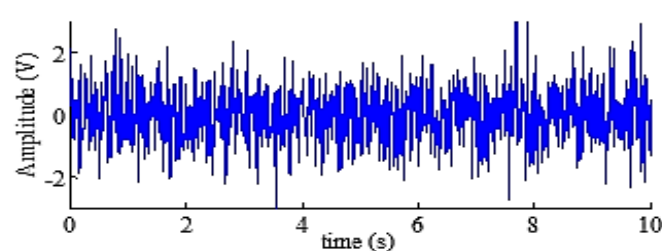

(a)

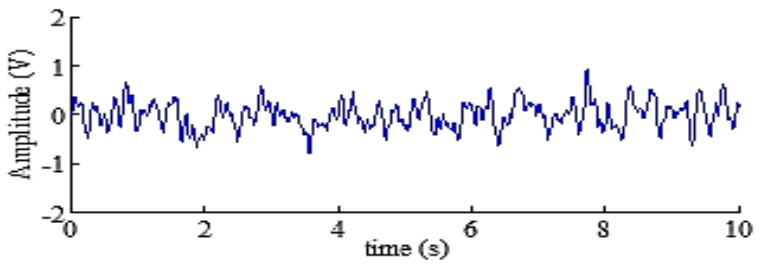

(b)

Figure 3. (a) Full-band limited white noise and (b) Sub-band limited white noise

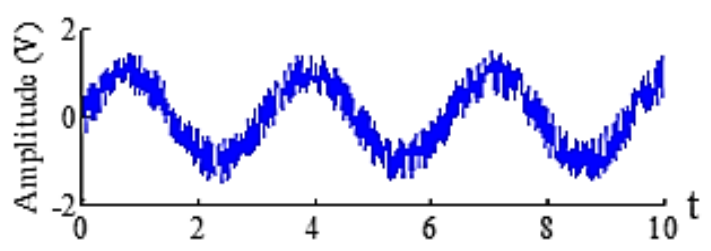

(a)

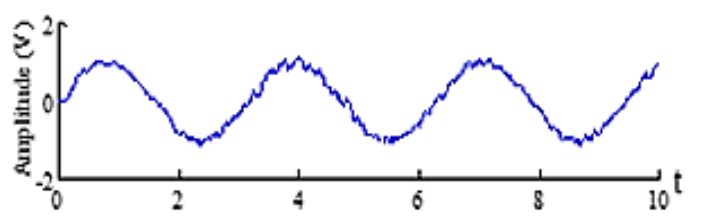

(c)

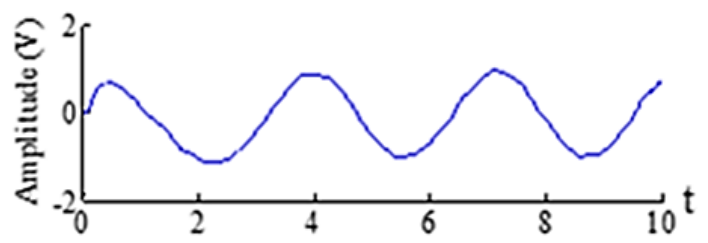

(b)

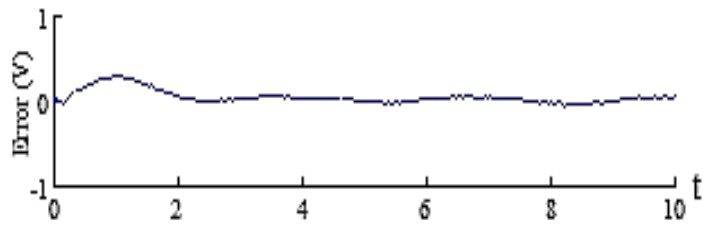

(d)

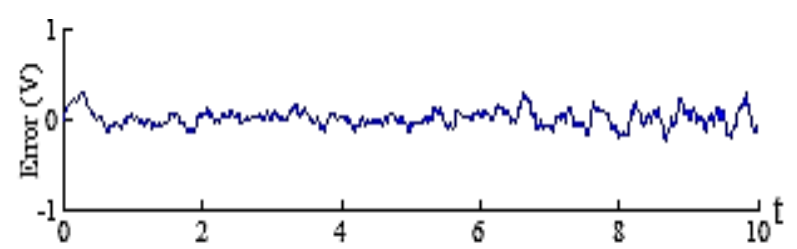

(e)

Figure 4. (a) The input signal distorted by band-limited white noise, (b) the output signal of ILMS noise canceller, (c) the output of LMS noise canceller, (d) the error signal of ILMS noise canceller and (e) the error signal of LMS noise canceller

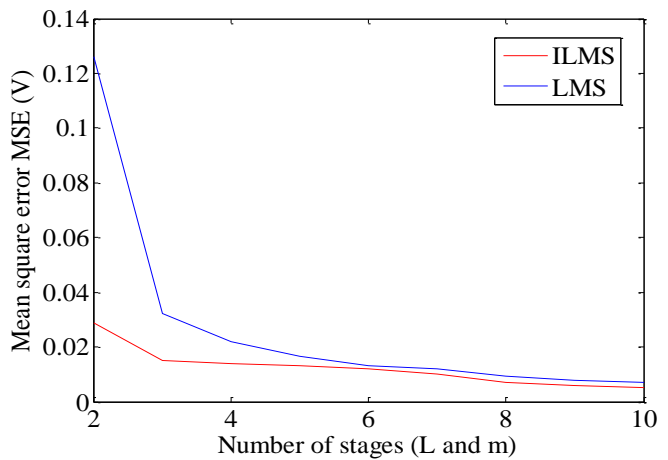

(a)

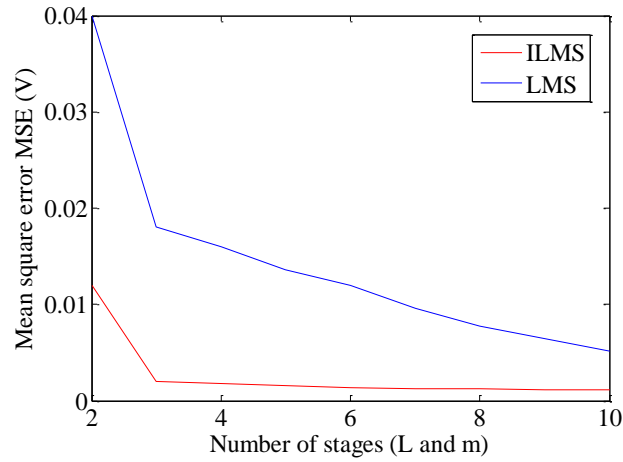

(b)

Figure 5. The comparison graph between the MSE of LMS adaptive noise canceller and MSE of ILMS adaptive noise canceller via the number of stages, (a) at $\mu=0.1$ and (b) at $\mu=0.2$ 
Table 1. Mean Square Error (MSE) Of the LMS Adaptive Canceller Via the Canceller Length (L)

\begin{tabular}{cc}
\hline $\mathrm{L}$ & MSE $(\mathrm{V})$ \\
\hline 2 & 0.1260 \\
3 & 0.0323 \\
4 & 0.0219 \\
5 & 0.0166 \\
6 & 0.0133 \\
7 & 0.0120 \\
8 & 0.0093 \\
9 & 0.008 \\
10 & 0.007 \\
\hline
\end{tabular}

Table 2. Mean Square Error (MSE) of the Ilms Adaptive Canceller Via the Number of Sub-Bands (M)

\begin{tabular}{cc}
\hline $\mathrm{m}$ & $\mathrm{MSE}(\mathrm{V})$ \\
\hline 2 & 0.0288 \\
3 & 0.015 \\
4 & 0.014 \\
5 & 0.013 \\
6 & 0.012 \\
7 & 0.01 \\
8 & 0.007 \\
9 & 0.006 \\
10 & 0.005 \\
\hline
\end{tabular}

\section{CONCLUSIONS}

In this work, the ILMS noise canceller using poly-phase digital filter bank has been developed to overcome the noisy adaptation problem in the LMS noise canceller due to noisy gradient of the instantaneous mean square error. In addition, the use of poly-phase filter bank for decomposition the full-band signal into sub-band signals will improve the convergence speed to the optimal output. An efficient implementation of 3-band uniform DFT poly-phase digital filter bank is used in this noise canceller. Hence, by using poly-phase digital filter bank with the LMS algorithm the misadjustment M of the LMS algorithm will be reduced and thus it will be allowed to increase the step size $\mu$ within its range to increase the convergence speed and overcoming the noisy adaptation problem.

\section{ACKNOWLEDGEMENTS}

This project is supported by Universiti Putra Malaysia.

\section{REFERENCES}

[1] S. M. Metev and V. P. Veiko, "Laser Assisted Microtechnology", 2nd ed., R. M. Osgood, Jr., Ed. Berlin, Germany: Springer-Verlag, 1998.

[2] J. Breckling, Ed., "The Analysis of Directional Time Series: Applications to Wind Speed and Direction", ser. Lecture Notes in Statistics. Berlin, Germany: Springer, 1989, vol. 61.

[3] S. Zhang, C. Zhu, J. K. O. Sin, and P. K. T. Mok, "A novel ultrathin elevated channel low-temperature poly-Si TFT," IEEE Electron Device Lett., vol. 20, pp. 569-571, Nov. 1999.

[4] M. Wegmuller, J. P. von der Weid, P. Oberson, and N. Gisin, "High resolution fiber distributed measurements with coherent OFDR,” in Proc. ECOC'00, 2000, paper 11.3.4, p. 109.

[5] R. E. Sorace, V. S. Reinhardt, and S. A. Vaughn, "High-speed digital-to-RF converter," U.S. Patent 5668842 , Sept. 16, 1997.

[6] Bendoumia, R., Kerkar, M., \& Bouzekkar, S. A. (2019). "Acoustic noise reduction by new sub-band forward symmetric adaptive decorrelation algorithms". Applied Acoustics, 152, 118-126.

[7] Wen, P., Zhang, J., Zhang, S., \& Li, D. (2019). "Augmented complex-valued normalized subband adaptive filter: Algorithm derivation and analysis". Journal of the Franklin Institute, 356(3), 1604-1622.

[8] So, H., \& Choi, J. W. (2019, April). "Subband Optimization and Filtering Technique for Practical Personal Audio Systems". In ICASSP 2019-2019 IEEE International Conference on Acoustics, Speech and Signal Processing (ICASSP) (pp. 8494-8498). IEEE.

[9] Dornean, I., Topa, M., Kirei, B. S., \& Neag, M. (2009, August). "Sub-band adaptive filtering for acoustic echo cancellation". In 2009 European Conference on Circuit Theory and Design (pp. 810-813). IEEE.

[10] Wada, T., Fang, J., Luo, F. L., Valkama, M., \& Tatu, S. O. (2015). "Advances in Digital Front-End and Software RF Processing: PartII". ZTECOMMUNICATIONS.

[11] Ni, J., \& Li, F. (2010). “A variable step-size matrix normalized subband adaptive filter". IEEE Transactions on Audio, Speech, and Language Processing, 18(6), 1290-1299.

[12] Seo, J. H., Jung, S. M., \& Park, P. (2016). "A diffusion subband adaptive filtering algorithm for distributed estimation using variable step size and new combination method based on the MSD”. Digital Signal Processing, 48, 361-369.

[13] Yu, Y., Zhao, H., \& Chen, B. (2016). "A new normalized subband adaptive filter algorithm with individual variable step sizes”. Circuits, Systems, and Signal Processing, 35(4), 1407-1418.

[14] Yang, F., Wu, M., Ji, P., \& Yang, J. (2012). “An improved multiband-structured subband adaptive filter algorithm”. IEEE Signal Processing Letters, 19(10), 647-650.

[15] Jeong, J. J., Kim, S. H., Koo, G., \& Kim, S. W. (2016). "Mean-square deviation analysis of multiband-structured subband adaptive filter algorithm”. IEEE Transactions on Signal Processing, 64(4), 985-994. (2002) The IEEE website. [Online]. Available: http://www.ieee.org/ 
[16] M. Shell. (2002) "IEEEtran homepage on CTAN". [Online]. Available: http://www.ctan.org/texarchive/macros/latex/contrib/supported/IEEEtran/

[17] George, N. V., \& Gonzalez, A. (2014). "Convex combination of nonlinear adaptive filters for active noise control". Applied Acoustics, 76, 157-161.

[18] Arenas-Garcia, J., Azpicueta-Ruiz, L. A., Silva, M. T., Nascimento, V. H., \& Sayed, A. H. (2016). "Combinations of adaptive filters: performance and convergence properties”. IEEE Signal Processing Magazine, 33(1), 120-140. FLEXChip Signal Processor (MC68175/D), Motorola, 1996.

[19] Jiang, J. Z., Shui, P. L., \& Zhang, Z. J. (2011). "Design of oversampled DFT-modulated filter banks via modified Newton's method". IET signal processing, 5(3), 271-280.

[20] "PDCA12-70 data sheet," Opto Speed SA, Mezzovico, Switzerland.

[21] A. Karnik, "Performance of TCP congestion control with rate feedback: TCP/ABR and rate adaptive TCP/IP," M. Eng. thesis, Indian Institute of Science, Bangalore, India, Jan. 1999.

[22] J. Padhye, V. Firoiu, and D. Towsley, "A stochastic model of TCP Reno congestion avoidance and control," Univ. of Massachusetts, Amherst, MA, CMPSCI Tech. Rep. 99-02, 1999.

[23] Sheikh, I. A., \& Pannu, N. K. (2018). "Review of Polyphase Filtering Technique in Signal Processing". 